\title{
CONTRIBUTIONS DE L'APPROCHE ERGONOMIQUE DE L'ACTIVITE À LA COMPRÉHENSION DU TRAVAIL DES ENSEIGNANTS DE FRANÇAIS
}

\section{NEGRÃO DOS SANTOS, Eliene do Socorro ${ }^{1}$ FARIAS, Aline Leontina Gonçalves ${ }^{2}$}

RÉSUMÉ: Cet article montre un aperçu des contributions de l'approche ergonomique de l'activité à la compréhension du travail des professeurs de français langue étrangère. Il s'agit d'une revue bibliografique, à partir de laquelle on souligne la prise en compte des aspects de l'action professorale qui n'étaient pas considérés avant dans les recherches traditionnelles en sciences de l'éducation (par exemple, les prescriptions, l'autoprescription, l'écart entre le prescrit et le réalisé, etc.). Ces aspects sont abordés dans les études ergonomiques qui s'intéressent à la compréhension de l'activité réelle (CLOT, 1999/2000). À partir d'un panorama d'études en sciences de l'éducation, on montre comment les recherches se sont

\footnotetext{
1 Diplômée en Lettres-Français à l'Université Fédérale du Pará, Brésil. E-mail : elienenegrao@outlook.com.

${ }^{2}$ Docteure, professeure et chercheuse à l'Université Fédérale du Pará, Brésil, Institut de Lettres et Communication - ILC, Faculté de Langues Étrangères Modernes - FALEM, Campus Belém. E-mail : alinefarias@ufpa.br.
} 
tournées de plus en plus vers le côté du professeur, en s'intéressant à ses manières de faire et d'agir en classe. Ces études ont fourni des fondements pour l'approche ergonomique de l'activité enseignante, dont on présente les notions et les concepts organisateurs ainsi que la méthode de l'autoconfrontation - centrée sur la production discursive des enseignants sur leur propre activité. Finalement, on discute quelques thèmes didactiques et ergonomiques importants de qui ont été mis en évidence par quelques recherches brésiliennes suivant l'approche ergonomique et utilisant l'autoconfrontation pour lancer un nouveau regard sur l'activité de l'enseignant de français.

MOTS-CLÉS : Ergonomie de l'activité, travail du professeur, enseignement du français langue étrangère.

\section{CONTRIBUIÇÕES DA ABORDAGEM ERGONÔMICA DA ATIVIDADE PARA A COMPREENSÃO DO TRABALHO DOS PROFESSORES DE FRANCÊS}

RESUMO: Este artigo fornece uma visão geral das contribuições da abordagem ergonômica da atividade para a compreensão do trabalho dos professores de francês língua estrangeira. Trata-se de uma revisão bibliográfica, a partir da qual enfatizamos a consideração de aspectos da ação professoral que não eram considerados antes nas pesquisas tradicionais nas ciências da educação (por exemplo, as prescrições, a autoprescrição, a distância entre o prescrito e o realizado, etc.). Esses aspectos são abordados nos estudos ergonômicos que se interessam pela compreensão da atividade real (CLOT, 1999/2006). A partir de um panorama de estudos nas ciências da educação, mostramos como as pesquisas se voltam cada vez mais para o lado do professor, interessando-se por suas maneiras de fazer e de agir em sala. Esses estudos forneceram fundamentos para a abordagem ergonômica da atividade docente, cujas noções e conceitos organizadores são apresentados neste artigo, assim como o método da autoconfrontação - centrado na produção discursiva dos professores sobre sua própria atividade. Finalmente, discutimos alguns temas didáticos e ergonômicos importantes colocados em evidência por algumas pesquisas brasileiras que seguiram a abordagem ergonômica e utilizaram a autoconfrontação para lançar um novo olhar sobre a atividade do professor de francês.

PALAVRAS-CHAVES: Ergonomia da atividade, trabalho do professor, ensino de francês língua estrangeira.

\section{INTRODUCTION}

L'enseignement a souvent provoqué des réflexions et des études en sciences de l'éducation. L'efficacité du professeur, reliée à sa façon d'enseigner, était dans la 
majorité des cas au centre de ces préoccupations. Comme le montre Amigues (2003, p. 1), « La façon d'appréhender la figure de l'enseignant a évolué avec le temps. Dans les années 1930-1950, aussi bien en France qu'aux États-Unis, l'approche positiviste prédominait dans la recherche des critères d'efficacité de l'action enseignante ».

Les recherches dans ce domaine soulignaient la responsabilité du professeur dans l'apprentissage des élèves, sans considérer les efforts déployés pour arriver à cette efficacité et aussi sans considérer le milieu classe. Dans cette optique, le cours devait provoquer un résultat donné chez l'élève pour que le professeur puisse être considéré un bon enseignant. Comme le remarque Saujat (2004, p. 7), «En effet, si la cible de l'action professorale est bien l'apprentissage des élèves, l'atteinte de cette cible n'est jamais directe ni immédiate : elle passe par les efforts des enseignants pour avoir une « classe qui tourne »».

À partir de ce contexte, on voit apparaître des recherches plus intéressées au travail réalisé par le professeur dans le domaine de l'approche ergonomique de l'activité. Ces études permettent de comprendre la complexité du travail du professeur, les réussites, les échecs, les intentions, les afflictions, etc. Le but de cet article c'est de comprendre les apports de l'approche ergonomique de l'activité enseignante à la compréhension du travail des enseignants de français langue étrangère.

Pour cela, cette étude est divisée en trois parties. Premièrement, on présente un panorama d'études en sciences de l'éducation pour montrer l'évolution de l'objet de recherche concernant l'enseignement. Deuxièmement, on présente l'approche ergonomique de l'activité et les notions qu'elle apporte pour lancer un nouveau regard sur l'enseignement compris et étudié en tant que travail, c'est-à-dire avec des apports des sciences du travail. Ensuite, on illustre des thèmes et des questions du travail réel (CLOT, 1999/2006) de l'enseignant de langues (surtout du FLE - français langue étrangère) mis en lumière par l'approche ergonomomique. Finalement, on discute brièvement les apports de l'approche ergonomique à la compréhension d'autres dimensions composantes et organisatrices de l'activité du professeur.

\section{PANORAMA D'ÉTUDES EN SCIENCES DE L'ÉDUCATION}


L'efficacité de l'enseignement a souvent été l'objet d'étude sur les pratiques éducatives. Selon Saujat (2004), ce thème - «important soit pour la recherche en éducation, soit pour les politiques scolaires et la formation des enseignants ${ }^{3}$ » (SAUJAT, 2004, p. 5) - a même servi de fil conducteur de la recherche sur l'enseignement depuis les premières études jusqu'à l'heure actuelle. Cet auteur présente alors un panorama de différentes approches en sciences de l'éducation qui ont alimenté la réflexion sur l'efficatité de l'enseignement et dont l'évolution conceptuelle a contribué à l'émergence du «travail du professeur» comme un nouvel objet de recherche.

Saujat (2004) divise ce panorama en différents paradigmes ou perspectives.

On résume quelques-un(e)s ci-dessous.

\section{LES ÉTUDES PROCESSUS-PRODUIT ${ }^{4}$}

Dans ces études, on conçoit le comportement du professeur en classe comme processus et l'apprentissage des élèves comme produit et on essaie d'évaluer l'efficacité de manière directe, c'est-à-dire en cherchant des relations directes entre l'agir du professeur et les indicateurs de l'efficacité perçus dans le produit. En d'autres mots, le cours devait avoir un résultat donné chez l'apprenant, soit positif, soit négatif, et c'était à partir de ce résultat que l'on pourrait identifier si l'agir de l'enseignant avait été efficace ou non. Mais il faut considérer que l'enseignant n'est pas l'unique responsable du déroulement de l'apprentissage des élèves, cela dépend de plusieurs facteurs.

D'après Saujat (2004: 7), en citant Clanet (2002) ${ }^{5}$, il y a eu quand même une considérable évolution dans ce modèle de recherche lorsqu'on a commencé à prendre en compte « les comportements des élèves en fonction des possibilités offertes par les

\footnotetext{
3 “importante tanto para as pesquisas em educação como para as políticas escolares e a formação de professore"” (SAUJAT, 2004, p. 5).

4 "Os estudos processo-produto" (SAUJAT, 2004, p. 7).

${ }^{5}$ Cité par Saujat (2004): CLANET, Joël. Gestion et organisation de de l'interaction maître-élèves. In P. Bressoux (Org). Les stratégies de l'enseignant en situation d'interaction. Note de synthèse pour Cognitique: Programme Ecole et Sciences Cognitives, 2002.
} 
professeurs ${ }^{6} »$. De cette manière, l'élève n'était plus considéré comme un simple recepteur dans le processus d'enseignement-apprentissage, et, par conséquent, l'efficacité n'était plus centrée sur le comportement du professeur. On commence à prendre en compte d'autres variantes médiatrices à mettre en relation avec le succès ou l'échec dans l'apprentissage.

Cependant, les études suivant la perspective processus-produit ont été critiquées. Selon Durand (1996) ${ }^{7}$, cité par Saujat (2004), les approches ne se basaient pas sur une méthode adéquate à l'établissement des relations de causalité comme elles avaient l'intention de faire et n'ont proposé aucune théorie de l'enseignement. Les études se centraient sur le comportement de l'enseignant en classe et les résultats qu'il provoquait dans l'apprentissage des élèves, mais il n'y avait pas de méthode suffisante pour expliquer les significations des comportements, puisque cela échappe aux yeux du chercheur. En outre, l'apprentissage - intitulé ici produit - ne dépend pas uniquement du comportement du professeur, il faudrait prendre en compte les conditions d'apprentissage, y compris le rôle de l'apprenant lui-même dans son processus d'appropriation. Par conséquent, il serait également problématique de concevoir l'apprentissage comme le seul paràmetre pour mesurer l'efficacité du travail enseignant.

Toutefois, les travaux du paradigme processus-produit ont contribué à mettre en relief l'enseignement comme objet de recherche.

\section{LE PARADIGME DE LA PENSÉE DES PROFESSEURS ${ }^{8}$}

Appelées au début teacher thinking, les recherches de ce paradigme, ont mis en place une approche cognitive de l'activité des professeurs. Les chercheurs ont essayé d'expliquer comment les professeurs faisaient pour résoudre les problèmes

6 "Os comportamentos dos alunos em função das possibilidades oferecidas pelos professores" (SAUJAT, 2004, p. 7).

${ }^{7}$ Cité par Saujat (2004): DURAND, Marc. L'enseignement en milieu scolaire. Paris : PUF, 1996.

8 “O Paradigma do pensamento dos professores" (SAUJAT, 2004, p. 9). 
rencontrés en classe et de comprendre leur façon de penser et réfléchir, la logique et la manière dont ils percevaient ces problèmes, la profession, la discipline.

Saujat (2004) distingue différentes approches dans ce paradigme. Par exemple, les études du courant appelé «les jugements du professeur et le processus de décision ${ }^{9} \gg($ SAUJAT, 2004, p. 10) ont opéré une rupture avec les études du type processus-produit. Ici, on met en relief l'activité cognitive du professeur. On ne pense pas seulement au produit, mais on fait aussi attention à la pensée du professeur, puisqu'on comprend finalement qu'elle va influencer tout le processus. Ainsi, on montre que ce n'est pas uniquement le produit qui est important dans le travail du professeur, mais tout ce qui fait partie du processus. Cependant, on s'est confrontré aux considérables défis méthodologiques. Par exemple, comment peut-on accéder à la cognition de chaque professeur? Les verbalisations ont été alors prises comme moyen d'accès aux processus de jugement et de décision réalisées par les enseignants. Toutefois, il restait le doute à propos de la valeur de ces verbalisations et de la partialité des reconstructions ou justifications reprenant les processus qui avaient été réellement mis en place lors du déroulement de l'action enseignante.

Les études du courant appelé «Les histoires personnelles de l'expérience ${ }^{10}$ » (SAUJAT, 2004, p. 13) se sont tournées vers l'expérience professionnelle de l'enseignant et étaient intéressées à la compréhension des différentes composantes de cette expérience. Ainsi, ces recherches se sont basées sur les méthodologies du type étude de cas, recherche de terrain et ont aussi fait recours à une collaboration narrative des participants avec les chercheurs. Cette approche a eu l'avantage de revitaliser la notion d'expérience, en s'intéressant à « la singularité de l'expérience professionnelle, dans ces dimensions subjectives et emotionnelles ${ }^{11} »($ SAUJAT, 2004, p. 13).

Saujat (2004) place encore dans ce paradigme la conception du «praticien réflexif » développée par Donald Schön $(1983,1987)^{12}$. Cette conception

\footnotetext{
9 "Os julgamentos do professor e o processo de decisão" (SAUJAT, 2004, p. 10).

10 "As histórias pessoais da experiência" (SAUJAT, 2004, p. 13).

11 "a singularidade da experiência profissional, em suas dimensões subjetivas e emocionais" (SAUJAT, 2004, p. 13).

${ }^{12}$ Cité par Saujat (2004): SCHÖN, Donald Alan. The reflective practitioner. New York: Basic Book, 1983.
} 
se base sur une approche empirique, selon laquelle la pensée professionnelle est comprise comme « une réflexion dans le cours de l'action ${ }^{13}$ »(SAUJAT, 2004, p. 13), sur l'action et la conduite dans un contexte donné. C'est donc une réflexion située, conçue comme un type de confrontation au réel. Pour les auteurs de ce courant, le savoir qui découle de ce raisonnement est difficile à formuler en mots puisqu'il se trouve incorporé. Il est, pourtant, «la source de l'efficacité de l'activité professionnelle ${ }^{14}$ » et peut «être représenté comme un dialogue des professionnels avec les problèmes des situations auxquels ils font face ${ }^{15} »$ (SAUJAT, 2004, p. 14).

Malgré ses avancées, la conception du praticien réflexif a été critiquée d'avoir pris un caractère normatif, c'est-à-dire d'avoir passé de la description à la proposition, voire imposition, des modèles normatifs pour orienter les programmes de formation.

\section{L'APPROCHE ÉCOLOGIQUE DES PROCESSUS INTERACTIFS ${ }^{16}$}

Quelques études situées dans cette approche ont proposé une description écologique de la classe, c'est-à-dire une description qui prenait en compte la diversité du travail enseignant par rapport aux situations et aux événements multiples et simultanés, parfois imprévisibles et demandant des prises de décision vites.

Altet (1991) $)^{17}$, cité par Saujat (2004 : 16), définit «l'enseignement comme un processus interactif, interpersonnel, intentionnel, finalisé par l'apprentissage des élèves ${ }^{18} \gg$. Selon l'auteur, ce processus est en rapport avec une culture professionnelle basée sur les six critères suivants :

a) la base de connaissances liée à l'activité professionnelle; b) une pratique adaptée dans une situation complexe; c) une capacité à

\footnotetext{
13 "uma reflexão no curso da ação" (SAUJAT, 2004, p. 13).

14 “a fonte de eficiência da atividade profissional” (SAUJAT, 2004, p. 14).

15 "pode ser representada como um diálogo dos profissionais com os problemas das situações com que se defrontam” (SAUJAT, 2004, p. 14).

16 "A abordagem ecológica dos processos interativos" (SAUJAT, 2004, p. 14).

${ }^{17}$ Cité par Saujat (2004): ALTET, Marguerite. Comment interagissent enseignant et élèves en classe? Revue Française de Pédagogie, 107, 1991.

18 "o ensino como um processo interativo, interpessoal, intencional, finalizado pela aprendizagem dos alunos" (SAUJAT, 2004, p. 16).
} 
rendre compte de ses propres compétences et actions; d) l'autonomie et la responsabilité personnelle dans l'exercice de ses compétences; e) l'adhésion à des normes collectives constituant une identité professionnelle; f) l'appartenance à un groupe qui développe des stratégies de promotion et de valorisation. (ALTET, 1994 apud SAUJAT, 2004, p. 16) ${ }^{19}$

Selon Saujat (2004, p. 16), ces critères ont l'intérêt de permettre la description des styles pédagogiques, qui impliquent trois dimensions interdépendantes: les styles personnel, rationnel et didactique. Les travaux centrés sur ce paradigme ont contribué à la prise en compte de la manière plus ou moins singulière dont chaque professeur, appartenant à une culture professionnelle donnée et partagée avec d'autres, arrive à gérer les situations de classe et à résoudre les problèmes qu'il y rencontre.

\section{LES RECHERCHES DANS LE DOMAINE DE LA DIDACTIQUE}

Les études dans le domaine de la didactique montrent d'abord un intérêt plus spécifiquement pour l'apprentissage des élèves. Amigues (2003) montre qu'il y a un caractère historique dans la centration sur l'apprentissage des élèves et explique que « les contraintes épistémologiques du savoir à transmettre et les actions des élèves laissent au second plan le travail réel de l'enseignant » (AMIGUES, 2003, p. 3). On a pu voir que la priorité de l'apprentissage des élèves a occupé une grande période des recherches en éducation. Cependant, cela commence à changer quand l'on commence à avoir plus de travaux qui considèrent l'activité enseignante dans son exécution : « les travaux portant sur l'activité de l'enseignant en situation de classe ont augmenté entre 1970 et 1980 (27 vs 99) mais dans l'ensemble des recherches, ces travaux restent peu nombreux $(5 \%)$ » (AMIGUES, 2003, p. 3). De cette façon, on a perçu un petit progrès

\footnotetext{
19 “a) a base de conhecimentos ligados ao agir profissional ; b) uma prática adaptada em situação complexa ; c) uma capacidade de dar conta de suas próprias competências e atos ; d) uma autonomia e uma responsabilidade pessoal no exercício de suas competências ; e) uma adesão a normas coletivas constitutivas da identidade profissional ; f) um pertencimento a um grupo que desenvolve estratégias de promoção e de valorização" (SAUJAT, 2004, p. 16).
} 
dans les études à propos des dimensions du travail enseignant, mais celui-ci n'était pas encore au centre.

Selon Saujat (2004), quelques travaux dans le domaine de la didactique des mathématiques et du français langue maternelle se sont tournés plus vers le côté du professeur, sa façon d'enseigner et de prendre des décisions en classe par rapport aux problèmes rencontrés et ses manières de les résoudre. L'intêrét pour le travail professoral commence alors à augmenter.

Ces études ont fourni des fondements pour de nouvelles recherches qui proposent un nouveau regard sur le travail enseignant, c'est-à-dire une approche ergonomique de l'activité enseignante. Dans cette nouvelle perspective, on remarque que l'efficacité de l'enseignement ne dépend pas seulement du professeur, elle fait partie de l'ensemble de l'activité enseignante. On présente cette approche plus en détails ci-dessous.

\section{L'ERGONOMIE DANS LES ÉTUDES DU TRAVAIL ENSEIGNANT}

L'approche ergonomique de l'activité enseignante est un nouveau domaine de recherche où l'on étudie l'enseignement comme travail. Cette approche, comme l'explique Souza-e-Silva (2004), s'établit à partir d'un apport fait à la tradition de l'ergonomie de langue française, qui a proposé un renversement de perspective. Ainsi, au lieu de s'intéresser à l'adaptation de l'homme au travail, l'ergonomie francophone s'occupe de l'adaptation des conditions de travail à l'homme, avec un souci de santé et développement professionnel et humain.

\section{APPROCHE ERGONOMIQUE DE L'ACTIVITÉ ENSEIGNANTE}

Cette nouvelle perspective d'étude est née au sein de l'équipe ERGAPE, acronyme d'Ergonomie de l'Activité de Professionnels de l'Éducation, à l'AixMarseille Université. Cette équipe réalise des recherches centrées sur trois différents objets : 
a) la prescription et son évolution ; b) les nouvelles organisations du travail (que ce soit dans les établissements scolaires ou la formation des enseignants) et c) l'étude des activités professionnelles en cours de (re)construction des personnels expérimentés comme des débutants. ${ }^{20}$

On essaye de mieux comprendre ce qui fait la complexité du travail enseignant. En effet, les points de vue sur le travail ont changé en donnant lieu à la nécessité d'un nouveau type d'étude, comme l'explique Amigues $(2009$, p. 12) : «Les sciences du travail ont montré depuis longtemps que tenter de caractériser le travail par son résultat ne permettait pas de connaître ce qui en fait son essence ; c'est même le meilleur moyen de soustraire cette dernière à l'analyse ». Dès lors, on ne peut pas mesurer l'efficacité d'un professionnel par l'apprentissage des élèves. En ce sens, d'autres auteurs présentent aussi leurs critiques et propositions : Faïta (2003) met en évidence les lacunes rencontrées dans la conception du travail, compris tout simplement en fonction de l'exécution ; Borghi (2006), à son tour, met en relief le besoin d'étudier la dimension du travail enseignant dans une approche ergonomique pour mieux comprendre l'activé du professeur.

Mais, c'est quoi l'ergonomie exactement? Le mot ergonomie vient du grec. Dans le sens étymologique, Ergon signifie «travail» et nomos signifie «normes, règles, lois ». Plus spécifiquement, pour Yvon et Saussez (2010, p. 9), ça veut dire «étude des lois du travail ».

L'ergonomie a eu lieu en Grande-Bretagne dans la seconde guerre mondiale et parallèlement en France, comme démontre Souza-e-Silva (2004). Ainsi, l'ergonomie a vu le jour en deux lieux différents et avec des conceptions et des préoccupations aussi différentes. En France, plus qu'en Grande-Bretagne, il est apparu les premières recherches ergonomiques, quand les chercheurs se sont intéressés au travail humain et à l'adaptation des conditions de travail à l'homme.

20 Source: Site de l'Aix-Marseille Université. Disponible sur : http://adef.univamu.fr/fr/\%C3\%A9quipe-ergape/pr\%C3\%A9sentation 
Martins (2007, p. 24) définit l'ergonomie comme « une étude spécifique du travail humain avec la finalité de l'améliorer, en cherchant de l'autonomie et des méthodes propres; elle donne priorité à l'analyse de l'activité ; vise comprendre le travail pour l'adapter à l'être humain ${ }^{21}{ }$. Pour Souza-e-Silva (2004), l'ergonomie fait partie d'un groupe de connaissances qu'englobe l'être humain, la pratique, la compréhension et la transformation du travail. Yvon et Saussez (2010), à leur tour, prennent en compte la santé des travailleurs en rapport aux conditions de travail. Cellelà est aussi une des préoccupations de l'approche ergonomique de l'activité enseignante, qui s'intéresse à l'amélioration des conditions de travail de l'enseignant. D'après Martins (2007), l'ergonomie étudie par exemple le comportement du professeur dans l'exercice de son métier, en relation avec le contexte où il est inséré. L'ergonomie mobilise quelques concepts importants pour mieux comprendre la construction du travail du professeur, par exemple le concept de prescription. En effet, l'ergonomie cherche à comprendre comment les prescriptions influencent ou modifient le travail enseignant. Comme le remarque Amigues (2003, p. 14),

\footnotetext{
Le point de vue adopté par l'approche ergonomique consiste à comprendre comment à partir des prescriptions qui lui sont faites, un professeur, non seulement utilise, mais refaçonne les moyens à sa disposition pour accrô̂tre, aussi bien "l'efficacité" que "l'efficience" de l'action : efficacité étant entendue ici comme orientée vers les élèves, mais aussi vers ses collègues qui doivent la reconnaître comme telle. (AMIGUES, 2003, p. 14).
}

On verra, par la suite, comment on conçoit le travail enseignant à partir des prescriptions.

\footnotetext{
21 "um estudo específico do trabalho humano com a finalidade de melhorá-lo, buscando autonomia e métodos próprios; prioriza a análise da atividade, entendendo o trabalhador como ator no processo de trabalho; visa compreender o trabalho para adaptá-lo ao ser humano" (MARTINS, 2007, p. 24).
} 


\section{PRESCRIPTIONS ${ }^{22}$, TÂCHE ET ACTIVITÉ DANS LE TRAVAIL ENSEIGNANT}

La prescription, entendue comme un ordre à être suivi, est à la base de l'activité enseignante. Dans un premier moment, les prescriptions étaient vues comme éloignées du travail réel du professeur. Comme le souligne Faïta (2003, p. 19), «les situations réelles contraignent l'enseignant à fournir des réponses à des questions que ces prescriptions ignorent, particulièrement tout ce qui "permet de faire ce qui est à faire", soit "prendre" une classe, "gérer" un groupe, etc..». Par contre, Amigues (2003) nous montre qu'elles sont très importantes: «Les prescriptions font évoluer le métier enseignant tout en ignorant ce qui en fait les ressorts.» (AMIGUES, 2003, p. 9). En tout cas, tel que le montre Amigues (2003), on ne peut pas les éloigner: « La prescription est non seulement à l'origine de l'action professorale, mais elle est consubstantielle de l'activité, telle que la conçoivent les ergonomes notamment dans ses dimensions collectives et individuelles, intersubjectives et subjectives.» (AMIGUES, 2003, p. 9).

La prescription peut aussi, d'après Amigues (2009), servir à organiser le milieu de travail du professeur. Dans la préparation de son activité enseignante, le professeur peut s'auto-prescrire des tâches qui ne sont pas formulées telles quelles dans la prescription. Comme l'explique Kayano (2005, p. 32), «Comme le terme l'indique, l'auto-prescription est le résultat d'une prescription et implique l'initiative de l'individu lui-même. ${ }^{23} \gg$. Martins (2007) comprend l'auto-prescription comme un ordre donné à l'individu par l'individu lui-même. En d'autres mots, s'auto-prescrire implique tout un travail de reconception et de connaissance de la prescription pour la transformer en tâches, selon ses besoins et de la manière la plus l'adéquate à la classe.

Dans l'exercice de son métier, le professeur est contraint tout le temps à suivre des prescriptions, cela arrive dès la $\operatorname{LDB}^{24}$ (au Brésil au niveau du gouvernement), les règlements (au niveau des institutions), et les règles du métier concernant le collectif

\footnotetext{
${ }^{22}$ Les prescriptions peuvent concerner, par exemple, les règlements de l'institution d'enseignement, les tâches proposées dans les livres et guides pédagogiques, des règles de conduite et des techniques formulées par le collectif de professeurs, etc.

23 "Como o termo indica, a auto-prescrição é a decorrência de uma prescrição e envolve a iniciativa do próprio indivíduo" (KAYANO, 2005, p. 32).

24 “Lei de Diretrizes e Bases". En français, on dirait : Loi de Directives et Bases.
} 
de travail (par exemple, la réorganisation du travail par les enseignants). C'est au professeur le rôle de mettre en rapport le travail prescrit et la manière dont il va ou peut le réaliser. Quelques auteurs partagent ce point de vue. Par exemple, Amigues (2003) attire l'attention sur la relation existante entre le prescripteur et le professeur par qui les prescriptions sont retravaillées. Saujat (2004) montre le rôle des prescriptions dans la réalisation de l'activité enseignante, elles ne servent pas seulement à déclencher l'action, mais sont aussi constitutives de l'activité. Kayano (2005), enfin, met en relief l'importance de la prescription dans la consolidation historique des manières de réaliser l'activité.

Dans ce cadre, il est encore important de distinguer entre le prescrit et le réalisé et souligner l'importance de cette distinction pour le travail enseignant. Borghi (2006) définit le travail prescrit comme tâche et le travail réalisé comme activité. Celles-ci sont deux notions importantes dans l'ergonomie. On peut donc remarquer la relation entre les dimensions prescrite et réalisée du travail. La prescription peut servir à conduire, organiser ou reformuler le travail du professeur. Elle joue un rôle important dans la conception de la tâche et de l'activité. Selon Saujat (2004, p. 39), «La tâche fait référence à ce qui doit être fait et peut être objectivement décrite en termes de conditions et d'objectif, de moyens (matériels, techniques...) utilisés par le sujet $^{25} \gg$; ou tout simplement, on peut dire que la tâche concerne les « prescriptions d'objectifs et de procédures ${ }^{26} \gg($ SOUZA-E-SILVA, 2004, p. 88).

Pour Saujat (2004), ce sont les tâches conçues à partir des prescriptions qui peuvent organiser le travail du professeur. Ce travail n'est pas défini par le propre travailleur, puisqu'il suit une hiérarchie déjà établie dans son milieu de travail. De cette manière, la tâche n'est pas individuelle, elle concerne tout le collectif de travailleurs de l'éducation. En d'autres mots, la tâche est toujours guidée par les prescriptions mais celles-ci montrent ce qu'on a à faire et pas exactement comment le faire. Par conséquent, cela pose des difficultés à la réalisation de la tâche. En fait, ces difficultés sont présentes dès la reformulation des prescriptions, moment où on essaye de mieux

\footnotetext{
25 "A tarefa refere-se ao que deve ser feito e pode ser objetivamente descrita em termos de condições e de objetivo, de meios (materiais, técnicos...) utilizados pelo sujeito" (SAUJAT, 2004, p. 39).

26 "Prescrições de objetivos e de procedimentos" (SOUZA-E-SILVA, 2004, p. 88).
} 
les adapter à l'exécution en salle de classe, mais aussi aux institutions où les enseignants sont insérés. Ceci est un problème auquel l'ergonomie s'intéresse. Comme le souligne Souza-e-Silva (2004, p. 90),

La difficulté rencontrée par l'enseignant en situation ne concerne pas seulement la nature des prescriptions, mais aussi leur mode de circulation dans le domaine de différents établissements scolaires, dont les formes d'organisation du travail de l'enseignant sont extrêmement variées et dont les effets sont vérifiés sans que leurs mécanismes sous-jacents soient explicités et expliqués, lacune qu'une analyse ergonomique du travail peut remplir. ${ }^{27}$

Selon Amigues (2009), l'espace entre le prescrit et le réalisé est constitué d'activité réalisée de façon individuelle ou collective. D'après Clot (1999/2006), entre le prescrit et le réalisé, il y a le réel de l'activité. On ne peut pas le voir effectivement, mais il est très important pour mieux comprendre l'activité.

Donc, l'analyse ergonomique s'intéresse aux manières de faire du professeur, aux connaissances qu'il mobilise pour accomplir les tâches qui lui sont prescrites. Dans l'approche ergonomique, ces connaissances mobilisées par l'enseignant sont aussi constitutives de l'activité. En ce sens, Saujat (2004) présente l'activité comme une chose qui ne peut pas être perçue par un observateur, elle correspond à ce que le professeur fait mentalement pour accomplir les tâches. D'après Clot et Faïta (2000), l'activité est conçue selon plusieurs aspects: les valeurs sociales, construites et partagées historiquement, mais aussi les valeurs individuelles issues de l'expérience personnelle. Ainsi, elle comprend plusieurs facteurs dans un même individu. Clot (1999/2006) met en relief le caractère psychologique de l'activité, pour lui, «L'activité

27 “A dificuldade com a qual depara o professor em situação não concerne apenas à natureza das prescrições, mas também ao seu modo de circulação no âmbito dos diferentes estabelecimentos escolares, cujas formas de organização do trabalho do professor são extremamente variadas e cujos efeitos são constatados sem que seus mecanismos subjacentes sejam explicitados e explicados, lacuna que uma análise ergonômica do trabalho pode preencher" (SOUZA-E-SILVA, 2004, p. 90). 
est une épreuve subjective où chacun fait face à soi-même et aux autres pour avoir la possibilité de pouvoir accomplir ce qu'il a à faire. ${ }^{28} \gg$ (CLOT, 1999/2006, p. 116).

Dans la réalisation du travail enseignant, l'activité ne se limite pas à ce que l'enseignant réalise en classe avec les élèves, elle recouvre plusieurs temporalités (AMIGUES, 2003). Pour Borghi (2006), l'activité réalisée par un professeur est plus complexe qu'il le semble, puisqu'il réalise différentes fonctions. Selon Amigues (2009), le professeur lui-même ne se rend pas compte du travail qu'il fait. Étant donné la complexité de l'activité du professeur, l'ergonomie veut mieux comprendre le travail enseignant à partir du point de vue du propre travailleur : «L'ergonomie aborde l'activité de travail comme élément central et structurant des composantes de la situation de travail. ${ }^{29}$ » (SOUZA-E-SILVA, 2004, p. 89). L'approche ergonomique de l'activité enseignante aborde les prescriptions, les (re)conceptions des tâches, le travail réalisé mais surtout le réel de l'activité. Comme l'observe Clot (1999/2006, p. 116), «Le réel de l'activité est aussi ce qui n'est pas fait, ce qui ne peut pas être fait, ce que l'on cherche à faire sans réussir - les échecs -, ce que l'on aurait voulu ou pu faire, ce qu'on pense ou ce qu'on rêve de pouvoir faire ailleurs. ${ }^{30}$ ».

C'est pour pouvoir accéder au point de vue des travailleurs et essayer de mieux comprendre le travail, au-delà de l'observable, c'est-à-dire pour avoir la chance de comprendre le réel de l'activité, qu'on a pensé à la méthode d'autoconfrontation, dont on parle ci-dessous.

\section{L'AUTOCONFRONTATION}

La méthode de l'autoconfrontation, grosso modo, consiste à déclencher le discours des travailleurs sur le travail en les confrontant à leurs propres actions en interaction avec le chercheur et aussi à un/des pair(s). L'ouverture de cet espace de

\footnotetext{
28 "A atividade é uma prova subjetiva em que cada um enfrenta a si mesmo e aos outros para ter uma oportunidade de conseguir realizar aquilo que tem a fazer” (CLOT, 1999/2006, p. 116).

29 "A ergonomia aborda a atividade de trabalho como elemento central e estruturante dos componentes da situação de trabalho" (SOUZA-E-SILVA, 2004, p. 89).

30 "O real da atividade é também aquilo que não se faz, aquilo que não se pode fazer, aquilo que se busca fazer sem conseguir - os fracassos -, aquilo que se teria querido ou podido fazer, aquilo que se pensa ou que se sonha poder fazer alhures" (CLOT, 1999/2006, p. 116).
} 
dialogue sur le travail permet aux travailleurs, d'après Faïta et Vieira (2003), de «mettre en chantier des manières de penser collectivement leur travail, entre l'image filmée de ce qu'ils ont fait et la mise en discours de ce qu'ils sont en train de faire. » (FAÏTA ; VIEIRA, 2003, p. 57).

L'autoconfrontation se compose de différentes phases dont les principales sont l'autoconfrontation simple et l'autoconfrontation croisée réalisées avec deux professionnels et le chercheur. L'autoconfrontation simple consiste dans le registre vidéo de l'activité enseignante de chaque professeur et après dans son interaction avec le chercheur face au film de son activité. À partir de la vidéo, c'est-à-dire son travail filmé, l'enseignant produit des commentaires à propos de son activité en favorisant l'émergence, c'est-à-dire la verbalisation des éléments jusqu'alors inobservables, cachés, implicites. L'autoconfrontation croisée consiste dans la confrontation des deux professeurs avec le chercheur, moment où l'un peut commenter l'activité de l'autre. Dans cette confrontation, il peut apparaître dans le discours des enseignants, entre autres choses, des similarités et des divergences sur les manières de faire et de penser.

Dans ce contexte, on a l'occasion de connaître quelques aspects importants du travail enseignant à partir des commentaires des propres enseignants. En effet, cette méthode permet de «prendre en compte les "données subjectives" qui témoignent des significations que les acteurs attribuent à leurs actions ». (AMIGUES, 2003, p. 13). Ainsi, le moment où le professeur est confronté au film de son activité, il a la possibilité de verbaliser des aspects significatifs de l'activité qu'il a réalisée, de la situation qu'il a vécue, et qui pourtant n'étaient pas connus ni observables jusqu'alors. De cette manière, l'enseignant met en lumière certaines questions de son travail, qui le poussent à réfléchir et justifier ses choix et ses décisions, tout en permettant de mieux comprendre son activité.

\section{LES CONTRIBUTIONS DE L'ERGONOMIE À LA COMPRÉHENSION DU TRAVAIL DE L'ENSEIGNANT DE FRANÇAIS}

Dans cette partie, on tente d'illustrer les contributions apportées à la compréhension du travail de l'enseignant de français par l'approche ergonomique de l'activité, surtout 
à partir de la mise en oeuvre de la méthode de l'autoconfrontation par deux recherches brésiliennes, celle de Farias (2011), qui a étudié la prise en compte et la gestion des prescriptions dans l'activité des professeurs stagiaires de français langue étrangère (désormais FLE), ainsi que l'existence d'un genre et des styles propres aux enseignants débutants ; et celle de Magalhães (2014), qui a exploité dans sa recherche le potentiel de l'autoconfrontation simple comme un instrument de formation de professeurs de FLE. Cette dernière auteure a employé des séquences d'autoconfrontation d'enseignants experimentés de langue française pour déclencher le débat et la réflexion parmi les étudiants stagiaires inscrits à la discipline de stage de la licence en Lettres mention FLE d'une université publique au Brésil.

Même s'il s'agit d'un petit nombre, ces deux recherches sont assez représentatives de cette nouvelle perspective d'étude du travail du professeur de français, dont on a envisagé de dresser un cadre plutôt illustratif et pas du tout exhaustif. Ces études ont fait émerger de nouveaux thèmes du métier de l'enseignant de FLE, surtout dans le rapport d'expérience des apprentis qui sont, selon nous, ceux qui peuvent être aidés davantage par les apports de l'ergonomie, à condition bien sûr que ces apports soient mobilisés pour et dans leur formation.

On verra que ces recherches ont permis de mettre en évidence, à partir de leur mise en discours, des éléments ou composantes important(e)s du travail réel des enseignants de français, dont en présentera quelques exemples ci-dessous : le dialogue avec la prescription, la réconception des tâches par les enseignants, la relation avec l'erreur.

\section{CONTESTATION ET ADAPTATION DE LA PRESCRIPTION}

L'analyse des dialogues des enseignants et chercheuses produits lors de la réalisation des interviews et des autoconfrontations a permis d'observer la partie constitutive du travail de réflexion dans l'activité des enseignants, c'est-à-dire le travail cognitif développé par le professeur dans et pour la réalisation des tâches. Il faut remarquer que cette réflexion ne pourrait être perçue qu'au moyen de sa 
matérialisation en discours, ou même par le biais de sa reconstruction dans la mise en mots des enseignants confrontés.

En général, les recherches ont mis en relief l'importance des prescriptions dans le métier enseignant, mais surtout le fait qu'elles sont retravaillées et réadaptées par les professeurs pour faire face aux contraintes du réel. Cela a pu être observé dans le discours des professeurs stagiaires sur leur propre travail. Dans la recherche de Farias (2011), on remarque bien que la professeure dialogue avec la prescription. Dans ce cas, l'enseignante n'est pas d'accord avec ce que la prescription propose et elle formule dans son discours une forme de contestation: «Bon, je pense que c'est une chose que je n'arrive pas à comprendre. Par exemple, dans le premier semestre, vous allez travailler les verbes de la première conjugaison, en général, mais les principaux, [rires] ils sont de la troisième conjugaison. [rires] ${ }^{31} \gg\left[\mathrm{R}^{32}\right]$ (FARIAS, 2011, p. 121). On perçoit, dans cet exemple, comment la professeure conteste et se met en désaccord avec les prescriptions. L'enseignante devait suivre le livre qui demande de travailler les verbes de la première conjugaison, mais elle est consciente que les verbes les plus importants, à son avis, sont de la troisième conjugaison. Pour nous, cette situation du travail enseignant, qui a émergé dans le discours de la professeure, illustre bien la remarque de Lousada (2006) à propos du rôle des prescriptions dans le métier enseignant : «Le travail du professeur est caractérisé et déterminé par plusieurs couches de prescriptions avec lesquelles l'enseignant dialogue, en les acceptant ou en s'y opposant. ${ }^{33} \gg$ (LOUSADA, 2006, p. 243).

Dans ce cas, on remarque que la professeure s'oppose à la prescription et dans la suite de son commentaire elle montre comment elle retravaille ce qu'elle rencontre dans les livres et reconçoit les prescriptions en fonction du contexte où elle est insérée :

\footnotetext{
31 "Bom, eu acho assim que é uma coisa que eu não consigo entender. Por exemplo, no primeiro semestre, você vai trabalhar os verbos da primeira conjugação, em geral, mas os principais, [risos] eles são da terceira conjugação. [risos]" (FARIAS, 2011, p. 121).

${ }^{32}$ Dans cette recherche, réalisée avec deux professeures, l'auteur a utilisé des codes pour les désigner$\mathrm{R}$ et $\mathrm{F}$ - sans pourtant les identifier.

33 "O trabalho do professor é caracterizado e determinado por várias camadas das prescrições com as quais o professor dialoga, concordando ou opondo-se a elas" (LOUSADA, 2006, p. 243).
} 
Aller, être, avoir, faire. Ces verbes, on ne peut pas quitter le premier semestre sans savoir comment les employer. Dans les deux examens, ils ont été employés, n'est-ce pas? [...] Alors, voilà, c'est là que j'ai ressenti le besoin, n'est-ce-pas. Plus loin, ils seraient exploités, mais je les présente depuis le début pour qu'on puisse s'y habituer. ${ }^{34}[\mathrm{R}]$ (FARIAS, 2011, p. 121)

Alors, on perçoit par là que, à partir de sa contestation du prescrit, la professeure reformule la demande du livre, la retravaille et l'adapte selon les besoins ressentis par elle.

\section{LA RECONCEPTION DES TÂCHES PAR LES ENSEIGNANTS}

La tâche, liée à la notion de prescription et aussi étudiée dans l'approche ergonomique de l'activité, est une autre composante importante du travail professoral. En effet, un des aspects couramment trouvés dans le travail du professeur, c'est non pas la simple exécution des tâches prescrites par d'autres, mais la reconception de celles-ci en tâches par le professeur pour lui-même et aussi pour ses élèves (AMIGUES, 2004).

Pour illustrer ce phénomène, on présente ci-après l'exemple d'une professeure qui montre dans son commentaire comment elle retravaille les prescriptions et les transforme en tâches pour elle-même. La prescription présentée par la professeure demande de faire écouter trois fois l'enregistrement sonore quand il s'agit d'une activité pour exercer la compréhension orale. Cepedant, on observe que la professeure avoue ne pas suivre cette prescription et elle explique la raison de le faire :

34 “Aller, être, avoir, faire. Esses verbos, não tem como sair do primeiro semestre sem saber empregar. Nas duas provas eles foram empregados, né. [...] Então, assim, aí foi que eu já senti a necessidade, né. Mais na frente é que eles iriam ser explorados, mas eu coloco logo no início pra que eles possam ir se acostumando." [R] (FARIAS, 2011, p. 121). 
Là moi, avant, juste un peu avant, j'ai répété trois fois, n'est-ce pas. Euh, parfois, je le répète beaucoup plus. Moi, je, je ne suis pas, ni en classe ni pour les examens, par exemple, un certain nombre de fois, ça doit être $\mathrm{N}$ fois. C'est que pour certains élèves, deux ou trois fois suffisent à me répondre, pour atteindre l'objectif que j'attends d'eux en tant qu'enseignante. Pour les autres non. J'ai donc toujours tendance à répéter et répéter, répéter et répéter. ${ }^{35}[\mathrm{R}]$ (FARIAS, 2011, p. 112)

Dans son commentaire, la professeure met en évidence sa préoccupation avec la compréhension du texte oral par tous les élèves. Ceci exige de l'enseignante tout un travail de reconception de la tâche en fonction des besoins de la classe, ce qui apparaît dans son discours comme une justification de l'adaptation réalisée par elle. C'est alors sa préoccupation qui détermine sa conduite et la réalisation de la tâche d'une certaine manière. Donc, la professeure fait écouter le dialogue plusieurs fois, contrairement aux trois écoutes qui lui étaient recommandées par la tâche initiale. Finalement, on doit remarquer qu'on ne peut pas savoir d'où vient cette prescription, puisque cela n'est pas exprimé dans le commentaire de l'enseignante. Toutefois, on peut faire l'hypothèse dont il s'agit d'une prescription présentée dans le manuel utilisé par elle ou dans l'école de langues où elle faisait son stage enseignant.

\section{LA RELATION AVEC L'ERREUR}

La question de l'erreur commise par l'enseignant lui-même a émergé comme un thème important de l'activité enseignante dans la recherche de Magalhães (2014), à partir des commentaires de deux professeurs stagiaires confrontés à l'activité et surtout au discours d'une professeure expérimentée, qui commente de manière assez naturelle le fait d'avoir commis une erreur.

\footnotetext{
35 “'Aí eu, antes né, um pouquinho antes, eu repeti três vezes, né. É, às vezes, eu repito muito mais. Eu, eu, eu não sigo, nem na aula e nem na prova, por exemplo, tantas vezes, tem que ser tantas vezes. Porque para alguns alunos duas ou três vezes é o suficiente pra me responder, pra chegar ao objetivo que eu como professora quero dele. Pra outros não. Então, eu sempre tenho uma tendência de repetir e repetir, repetir e repetir" [R] (FARIAS, 2011, p. 112).
} 
Au tout début de la discussion, on a remarqué que l'emploi erroné de la langue étrangère - objet d'enseignement et d'apprentissage - peut causer une insatisfaction assez importante et parfois troublante chez les professeurs débutants. Cependant, dans la suite du dialogue, on verra que le fait de savoir que les enseignants expérimentés se trompent parfois et se corrigent devant la classe quand cela arrive crée un débat positif chez les enseignants débutants.

Tout d'abord, le commentaire de l'enseignante expérimentée semble avoir encouragé l'un des professeurs stagiaires à raconter son expérience quand il a également fait une erreur devant la classe dans son premier jour de cours. Il raconte alors comment il s'est senti:

Le premier jour de cours euh +++ que j'ai eu dans le groupe [A2 fait référence à son groupe d'étudiants du Centre de langues $\left.{ }^{36}\right]$, j'ai fait une erreur concernant un verbe et +++ quand un étudiant m'a corrigé, moi dans (XXXX). Oh ! j'ai tremblé. (rires). [...] et c'était une chose tellement stupide [...] je sais que je savais la forme correcte, mais à ce moment-là, par tension, parce que c'était le premier jour +, ça c'est arrivé. ${ }^{37}$ [A2] ${ }^{38}$ (MAGALHÃES, 2014, p. 134).

Dans ce commentaire, l'erreur est mentionnée comme un élément négatif dans l'activité du professeur. On peut percevoir que l'enseignant débutant se sent agacé à cause de l'erreur qu'il a commise, surtout par le fait qu'il s'agissait d'une erreur qui ne posait pas de difficulté, comme il le remarque dans ce passage « et c'était une chose tellement stupide ». La situation devient encore plus embarrassante pour

\footnotetext{
${ }^{36}$ Il s'agissait d'un Centre de langues lié à l'université et destiné aux étudiants de la licence en langues étrangères, où les étudiants qui réussissaient les examens didactiques pouvaient réaliser leur stage enseignant.

37 "No primeiro dia de aula é +++ que eu tive no grupo [A2 refere-se ao seu grupo de alunos do Núcleo de Línguas], eu cometi um erro em relação a um verbo e +++ quando um aluno me corrigiu, eu na (XXXX). Poxa, eu fiquei trêmulo. (risos).[...] e era uma coisa tão boba [...] eu sei que eu sabia o certo, mas naquele momento, por nervosismo, por ser o primeiro dia + , aconteceu." [A2] (MAGALHÃES, 2014, p. 134).

${ }^{38}$ Il s'agit de professeurs débutants confrontés à l'ativité et aux commentaires de professeurs expérimentés de français.
} 
l'enseignant au moment où son erreur est perçue et corrigée par son élève : « quand un étudiant m'a corrigé, moi dans (XXXX). Oh ! j'ai tremblé ». À cause de cela, le professeur débutant se sent un peu angoissé. L'espace et le débat ouverts par l'autoconfrontation ont été importants pour faire connaître le sentiment du jeune professeur dans la réalisation de son travail, dans ce cas, la souffrance provoquée chez lui par une erreur.

Par contre, dans l'évolution du dialogue, on arrive à un moment où l'on peut voir le côté positif du débat. Il s'agit du commentaire de l'autre professeure stagiaire, qui révèle une approche - et on pourrait même dire une appropriation - positive de ce qui avait été dit par la professeure expérimentée:

Je ne pense pas que ce soit si compliqué. Puisqu'elle est une enseignante expérimentée et montre l'humilité de dire qu'elle ne sait pas tout, que si elle a quelque problème +++ , qu'elle s'est trompée dans l'enseignement d'un certain contenu dans une classe, dans l'autre elle va ++ corriger. C'est génial pour nous qui débutons et aussi n'avons pas à nous blâmer pour les erreurs que nous faisons. Donc, il faut essayer d'améliorer, tel qu'elle le fait, n'est-ce pas $?^{39}$ [A3] (MAGALHÃES, 2014, p. 136)

Dans cette partie, on voit comment la professeure stagiaire comprend la question de l'erreur différemment de son collègue. Il semble que, pour elle, l'erreur c'est une chose plus naturelle: «je ne pense pas que ce soit si compliqué ». On remarque que le cas de la professeure expérimentée qui se corrige le cours suivant, si elle se rend compte d'avoir commis une erreur, devient un bon exemple et rassure les professeurs débutants à propos du fait que l'erreur fait partie du processus d'enseignement-apprentissage. Donc, il ne faut pas rester dans l'inquiétude ou encore comme la stagiaire a remarqué : «[nous] n'avons pas à nous blâmer » si cela arrive.

\footnotetext{
39 "Eu não acho assim tão complicado. Porque é uma professora experiente e tá mostrando a humildade de dizer que ela não sabe tudo, que se ela tiver algum problema +++, que ela ensinou algum conteúdo errado numa aula, na outra ela vai ++ corrigir. Isso é ótimo pra gente que tá começando e também não tem que se culpar pelos erros que a gente tem. Então, tem que tentar melhorar, assim como ela faz, né?" [A3] (MAGALHÃES, 2014, p. 136).
} 
Elle comprend que ce qu'il faut c'est d'essayer toujours de progresser dans le métier comme la professeure expérimentée le fait et aussi avoir conscience qu'on a toujours des choses à apprendre : « Donc, il faut essayer d'améliorer, tel comme elle le fait ».

On voit, qu'à partir de l'autoconfrontation, on a l'occasion de discuter à propos des sentiments éprouvés par les professeurs dans leur l'activité et on a aussi la possibilité d'aider les professeurs stagiaires à rencontrer des solutions positives pour les problèmes qu'ils rencontrent en classe et aussi les aider à surmonter les souffrances comme celle provoquée par une éventuelle erreur. Dans cette optique, Magalhães (2014) souligne que :

Travailler, selon nous, c'est aussi prendre des décisions, faire des choix, errer et corriger l'erreur, organiser et réorganiser ce qui doit être fait, faire des inférences et des autoprescriptions, conjecturer, savoir gérer les variabilités qui se présentent, transformer et être transformé, enfin, travailler va bien au-delà d'une simple et mécanique production matérielle. ${ }^{40}$ (MAGALHÃES, 2014, p. 52).

Finalement, on peut remarquer que l'inquiétude face à l'erreur, c'est un thème qui était implicitement partagé par les enseignants débutants et il apparaît comme une partie importante de leur activité professorale. Toutefois, il n'était pas considéré avant. C'est grâce à l'approche ergonomique et au dialogue centré sur les commentaires d'une professeure expérimentée à propos de son erreur que les enseignants débutants ont pu partager explicitement ce thème, parfois si embarrassant pour les débutants ; et c'est dans le dialogue, c'est-à-dire dans le développement conjoint de la discussion de ce thème, qu'ils arrivent à concevoir différemment la situation et à construire, dans leur discours, une nouvelle relation avec l'erreur.

\section{CONCLUSION}

40 "Trabalhar, no nosso entender, é também tomar decisões, fazer escolhas, errar e corrigir o erro, organizar e reorganizar o que deve ser feito, fazer inferências e autoprescrições, conjecturar, saber gerir as variabilidades que se apresentam, transformar e ser transformado, enfim, trabalhar vai muito além de uma simples e mecânica produção material.” (MAGALHÃES, 2014, p. 52). 
Cet article a essayé de montrer, dans un premier temps et de manière plus générale, comment l'intérêt pour le travail enseignant a augmenté au fil du temps. Ensuite, on a essayé d'illustrer, plus spécifiquement, les contributions apportées par l'approche ergonomique à la compréhension de l'activité des enseignants de français, surtout des enseignants débutants.

On a pu remarquer une évolution dans les recherches en éducation. D’abord, on s'intéressait surtout à l'apprentissage des élèves. Ensuite, les recherches dans le domaine de la didactique ont montré un certain virage vers le travail professoral, quand on a commencé à prendre en compte d'autres variables composantes de l'activité du professeur que les seules manières de faire causalement liées à l'efficacité dans l'apprentissage.

Dans la suite, depuis le début des années 2000, l'approche ergonomique de l'activité enseignante propose d'étudier le travail professoral dans une nouvelle perspective, façonnée à partir d'un emprunt conceptuel et méthodologique aux sciences du travail. Autrement dit, ayant l'intérêt de comprendre «l'enseignement comme travail » (MACHADO, 2004), on a mobilisé des concepts tels que prescription, autoprescription, tâche, activité, travail prescrit, travail réalisé, entre autres, ainsi que des méthodologies conçues dans le but d'analyser l'activité enseignante, à l'exemple de la méthodologique de l'autoconfrontation. Ainsi outillé, on entreprend des recherches dont le but général c'est de mieux comprendre l'activité du professeur en essayant de s'approcher petit à petit de sa complexité.

Finalement, on a essayé d'illustrer comment quelques composantes importantes et jusqu'alors implicites de l'activité de l'enseignant de français ont pu émerger dans deux recherches situées dans l'approche ergonomique et utilisant la méthodologie de l'autoconfrontation, contribuant selon nous à comprendre à nouveaux frais cette activité.

Grâce à cette approche, on a pu donner plus de visibilité au travail du professeur, aux aspects qui n'étaient pas considérés avant, comme le travail qu'il réalise en classe, les problèmes rencontrés et ce qu'il fait pour les résoudre. De cette façon, on peut mieux comprendre l'activité enseignante à partir des commentaires 
produits par le professeur sur sa propre activité et ainsi on rend visible et discutable les réflexions du professeur dans son agir, par exemple le dilemme de la traduction, les angoisses face à l'erreur, etc. Enfin on peut voir l'importance de cette nouvelle perspective d'étude et les apports qu'elle offre pour lancer un nouveau regard sur le travail enseignant ce qui peut aider le professeur à mieux connaître son activité et aussi lui-même.

Cette étude nous a permis de comprendre que l'activité qu'un enseignant réalise est assez grande et multiple et que d'habitude le professeur ne se rend même pas compte du travail complexe qu'il réalise. On a pu constater que les notions et concepts de l'approche ergonomique favorisent la compréhension des dimensions implicites de la pratique enseignante et que celles-ci peuvent devenir observables et discutables grâce à la méthodologie de l'autoconfrontation qui ouvre l'espace pour que les professeurs puissent eux-mêmes mettre en discours leur propre travail. On croit que le fait de discuter l'activité à partir de son enregistrement vidéo permet aussi aux professeurs, surtout les professeurs stagiaires, de mieux comprendre leur pratique. Par exemple, on a pu voir comment les stagiaires confrontés au discours d'une professeure expérimentée ont pu se rendre compte de ce qu'ils ressentent comme problème est souvent partagé par le collectif des enseignants, ce qui les a encouragé à construire et reconstruire discursivement leur relation avec l'erreur.

Enfin, on remarque le potentiel de cette approche pour une compréhension plus large de la pratique des professeurs voire sa transformation, puisque la compréhension de la complexité de l'activité qu'on réalise peut nous pousser à réfléchir et à élaborer collectivement des solutions pour nos détresses.

\section{REFERÊNCIAS BIBLIOGRÁFICAS}

AMIGUES, René. Le travail enseignant: prescriptions et dimensions collectives de l'activité. Les Sciences de l'éducation Pour l'Ėre nouvelle. vol. 42, n², 2009. pp. 11- 
26. Disponível em: <http://www.cairn.info/revue-les-sciences-de-l-education-pour-lere-nouvelle-2009-2-page-11.htm>. Acesso em: 16 nov. 2015.

. Trabalho do professor e trabalho de ensino. Tradução de Anna Rachel Machado. In: MACHADO, Anna Rachel (Org.). O ensino como trabalho: uma abordagem discursiva. Londrina: Eduel, 2004, pp. 35-53.

Pour une approche ergonomique de l'activité enseignante. Skholê, horssérie 1, 2003. pp. 5-16. Disponível em:

F<http://www.aixmrs.iufm.fr/recherche/publ/skhole/pdf/03.HS1.5-16.pdf >. Acesso em: 04 set. 2011.

BORGHI, Carmen Ilma Lima Belincanta. A configuração do trabalho real do professor de língua inglesa em seu próprio dizer. 2006. Dissertação. (Mestrado em Estudos da Linguagem) - UEL, 2006.

CLOT, Yves. A função psicológica do trabalho. Tradução de Adail Sobral. Petrópolis, RJ: Vozes, 1999/2006.

CLOT, Yves; FAÏTA, Daniel. Genres et styles en analyse du travail: concepts et méthodes. Travailler, 2000, pp. 7-42.

FAÏTA, Daniel. Apports des sciences du travail à l'analyse de l'activité. Skholê, horssérie 1, 2003, pp. 17-23.

FAÏTA, Daniel; VIEIRA, Marcos. Réflexions méthodologiques sur l'autoconfrontation croisée. Skholê, hors-série 1, 2003, pp. 57-68.

FARIAS, Aline Leontina Gonçalves. Atividade docente de estagiários de francês: prescrições, gênero e estilo. 2011. Dissertação. (Mestrado) - Curso de Mestrado Acadêmico em Linguística Aplicada) - Universidade Estadual do Ceará, Fortaleza, 2011. KAYANO, Letícia Moreira Dias. A relação prescrito / real em sala de aula. 2005. Dissertação. (Mestrado em Linguística Aplicada e Estudos da Linguagem) - PUC-SP, 2005.

LOUSADA, Eliane Gouvêa. Entre trabalho prescrito e realizado: um espaço para a emergência do trabalho real do professor. 2006. Tese. (Doutorado) - Programa de Pósgraduação em Linguística Aplicada e Estudos da Linguagem, Pontífice Universidade Católica de São Paulo, 2006. 
MAGALHÃES, Elisandra Maria. Autoconfrontação Simples (ACS): um instrumento a serviço da formação profissional de futuros professores de francês língua estrangeira (FLE). 2014. Dissertação. (Mestrado) - Programa de Pós-graduação em Linguística Aplicada, Universidade Estadual do Ceará, Fortaleza, 2014.

MARTINS, Eliane Provate Queiróz. O ensino de inglês em uma franquia de idiomas: uma análise do trabalho prescrito e do real. 2007. Dissertação. (Mestrado em Estudos da Linguagem) - Universidade Estadual de Londrina, 2007.

SAUJAT, Frédéric. O trabalho do professor nas pesquisas em educação: um panorama. In: MACHADO, Anna Rachel. (Org.). O ensino como trabalho: uma abordagem discursiva. Londrina: Eduel, 2004, pp. 5-34.

Educating the reflective practitioner. San Francisco: Jossey-Bass, 1987.

SOUZA-E-SILVA, Maria Cecília Pérez de. O ensino como trabalho. In: MACHADO, Anna Rachel. (Org.). O ensino como trabalho: uma abordagem discursiva. Londrina: Eduel, 2004, pp. 81-104.

YVON, Frédéric; SAUSSEZ, Frédéric. Analyser l'activité enseignante - Des outils méthodologiques et théoriques pour l'intervention et la formation. Québec: Les Presses de l'Universite Laval, 2010, pp. 9-16. 